\title{
Succinate: a metabolic signal in inflammation
}

\author{
Evanna Mills and Luke A.J. O'Neill
}

School of Biochemistry and Immunology, Trinity Biomedical Sciences Institute, Trinity College Dublin, Dublin 2, Ireland

\begin{abstract}
Succinate is an intermediate of the tricarboxylic acid (TCA) cycle, and plays a crucial role in adenosine triphosphate (ATP) generation in mitochondria. Recently, new roles for succinate outside metabolism have emerged. Succinate stabilizes the transcription factor hypoxia-inducible factor-1 $\alpha$ (HIF-1 $\alpha$ ) in specific tumors and in activated macrophages, and stimulates dendritic cells via its receptor succinate receptor 1 . Furthermore, succinate has been shown to post-translationally modify proteins. This expanding repertoire of functions for succinate suggests a broader role in cellular activation. We review the new roles of succinate and draw parallels to other metabolites such as $\mathrm{NAD}^{+}$and citrate whose roles have expanded beyond metabolism and into signaling.
\end{abstract}

Metabolic alterations influence the immune response The immune system acts as an internal shield defending against invading pathogens and is made up of an innate system, including macrophages, neutrophils, and dendritic cells (DCs), and an adaptive system composed of T and B lymphocytes. Cells of the innate immune system recognize pathogen-associated molecular patterns (PAMPs) via pattern recognition receptors (PRRs), such as Toll-like receptors (TLRs), present on their cell surface and in the cytosol [1]. Once activated, innate immune cells can initiate adaptive immunity to fight infection further and to generate immunological memory. In resting conditions these cells are relatively inactive; however, upon recognition of foreign material they have the ability to respond rapidly, producing a wide array of mediators such as cytokines, chemokines, and antimicrobial peptides to clear the infection.

Such rapid induction of immunity represents a significant metabolic demand. In recent years it has become evident that immune cells have the ability to shift their metabolism under varying conditions and that this is essential for proper immune function [2]. Stimulation of DCs and macrophages can result in a decrease in oxidative phosphorylation, which is normally employed under resting conditions, with a concomitant increase in glycolysis and the pentose-phosphate pathway $[3,4]$. This switch to

Corresponding author: O’Neill, L.A.J. (laoneill@tcd.ie).

Keywords: metabolism; succinate; inflammation; SUCNR1; HIF-1 $\alpha$; succinylation.

0962-8924/\$ - see front matter

(c) 2013 Elsevier Ltd. All rights reserved. http://dx.doi.org/10.1016/j.tcb.2013.11.008 glycolysis rapidly generates ATP to maintain mitochondrial membrane potential and energy homeostasis, ultimately ensuring cell viability [5]. It also provides biosynthetic precursors required for proliferating cells and for cells with a prodigious biosynthetic capacity, such as macrophages when they are activated to produce cytokines. This altered metabolism is similar to the Warburg effect (aerobic glycolysis) observed in tumor cells [6] (Figure 1, Box 1). Indeed, the metabolic sensor HIF-1 $\alpha$ can determine the growth and fate of both tumor cells and immune cells. For example, HIF-1 $\alpha$ activation favors differentiation of T lymphocytes into proinflammatory Th17 cells and attenuates regulatory $\mathrm{T}$ cell development [7]. Importantly, as well as global changes in metabolism that occur in activated immune cells, individual metabolites, including succinate, citrate, and $\mathrm{NAD}^{+}$, have been demonstrated to possess signaling capacity and to influence immunity $[4,8,9]$. These somewhat surprising findings allow for metabolic changes occurring in inflammatory cells to serve as signals to which cells respond and can contribute to the inflammatory process (Figure 2).

In this review we summarize recent findings related to these metabolic changes, focusing on citrate, $\mathrm{NAD}^{+}$, and succinate. The changes in succinate are of particular interest given the recent findings in relation to downstream targets for succinate, including HIF- $1 \alpha$, the signaling receptor succinate-receptor 1 (formally termed G proteincoupled receptor 91), and the post-translational modification (PTM) of proteins by succinylation. We discuss the role played by these events in immunity. These expanding roles for metabolites suggest that TCA cycle intermediates may represent a novel class of regulators of inflammation acting as key signals in multiple biological processes.

\section{Citrate transportation regulates inflammatory responses}

An increase in metabolite transporters in mitochondria, in addition to various metabolites, was observed in a metabolic screen and microarray analysis of macrophages treated with lipopolysaccharide (LPS), a component of the outer membrane of Gram-negative bacteria [4]. This finding suggests that the transport of particular mediators from the mitochondria to the cytosol is required following LPS stimulation in macrophages. Among the various metabolic transporters, the mitochondrial citrate carrier (CIC) was found to be increased following LPS stimulation [10], probably due to activation of one or both of the two nuclear factor $\kappa \mathrm{B}(\mathrm{NF}-\kappa \mathrm{B})$ sites contained in the CIC promoter. Furthermore, knockdown of CIC with small interfering 


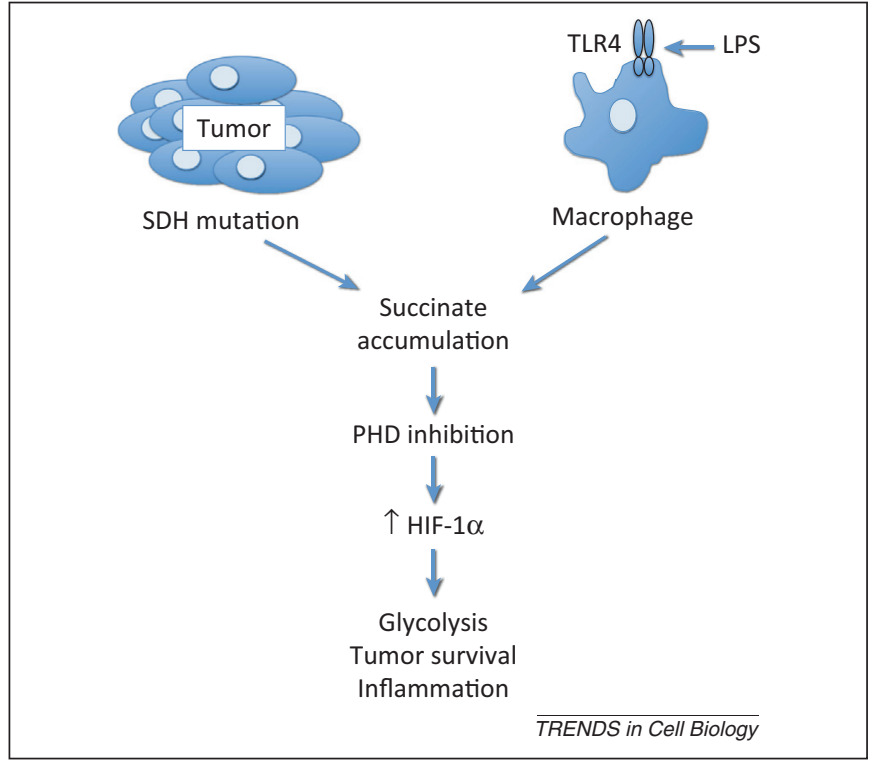

Figure 1. Parallels between tumors and lipopolysaccharide (LPS)-activated macrophages in relation to succinate. Both macrophages treated with LPS and tumors with succinate dehydrogenase (SDH) mutations display elevated levels of succinate. Succinate stabilizes hypoxia-inducible factor- $1 \alpha$ (HIF- $\alpha$ ) by inhibiting prolyl hydroxylase domain (PHD) enzyme activity. Stabilized HIF- $\alpha$ binds to HIF response elements (HREs) in target genes, including those encoding glycolytic enzymes, angiogenic factors, and the inflammatory cytokine interleukin $1 \beta$ (IL-1 $\beta$ ), thereby enhancing tumor survival and growth, and exacerbating inflammation. This parallel HIF- $1 \alpha$ activation represents another link between inflammation and cancer. TLR4, Toll-like receptor 4 .

RNA (siRNA), or by the use of an inhibitor, benzene-1,2,3tricarboxylate, impaired the production of reactive oxygen species (ROS), nitric oxide (NO), and prostaglandins (PGs) in macrophages treated with LPS, suggesting that CIC is required for the induction of these mediators. CIC is responsible for the transport of citrate from the mitochondria, where it is produced as part of the TCA cycle, to the cytosol. Citrate is needed for fatty acid biosynthesis and, once in the cytosol, is cleaved by citrate lyase into acetylCoA and oxaloacetate, precursors for NO, ROS, and arachidonic acid (which is used to generate PGs). Although studies that demonstrate a direct role for citrate in inflammation are currently lacking, an elevation in citrate and a decrease in isocitrate was observed in LPS-activated macrophages [4], further supporting the role of this metabolite in LPS-induced responses. Citrate is therefore acting as a signal to produce key reactive oxygen intermediates for host defense and inflammation, as well as PGs, which are important mediators of inflammation.

\section{$\mathrm{NAD}^{+}$and sirtuins as regulators of inflammation}

Another well-known metabolite, $\mathrm{NAD}^{+}$, is emerging as a regulator of inflammation by acting through sirtuins. The sirtuins make up a family of $\mathrm{NAD}^{+}$-dependent deacetylases which influence chromatin structure and protein function by removing acetyl groups [11]. Owing to their dependence on $\mathrm{NAD}^{+}$, they act as sensors of the energy status of the cell - oxidative metabolism determines the ratio of $\mathrm{NAD}^{+}$to $\mathrm{NADH}$, whereas glycolysis promotes a switch towards $\mathrm{NADH}$. Activation of these enzymes has been shown to promote anti-inflammatory responses. Among the seven sirtuins (Sirt1-7) found in mammals, Sirt1 has been shown

\section{Box 1. The relationship between succinate and HIF-1 $\alpha$ in} cancer

Mutations in metabolic enzymes are sufficient to initiate, as well as exacerbate, tumor development. Importantly, mutations in the gene encoding succinate dehydrogenase $(S D H)$, the enzyme responsible for the conversion of succinate to fumarate, were implicated in several cancers, and SDH is now regarded as a tumor suppressor [42]. SDH mutations result in the accumulation of succinate and subsequent HIF-1 $\alpha$ stabilization [43], thereby providing a growth advantage for tumors. HIF-1 is a transcriptional regulator in the switch to glycolysis [22] and has been shown to be an important target for succinate. It binds to HIF response elements (HREs) in target genes and is responsible for increasing the expression of most glycolytic enzymes. HIF-1 $\alpha$ contributes to tumorigenesis by increasing glucose metabolism, generating intermediates for proliferation, and enhancing angiogenesis. HIF is tightly regulated by PHD enzymes, a class of dioxygenases that use oxygen derived from the conversion of $\alpha$-ketoglutarate $(\alpha-K G)$ to succinate to hydroxylate HIF-1 $\alpha$. This primes it for ubiquitination by E3 ubiquitin ligase and subsequently targets it for proteasomal degradation [44]. In tumor cells succinate has been shown to inhibit PHD activity via product inhibition and to increase HIF- $1 \alpha$ stabilization [43]. This important finding was the first indication that succinate is capable of acting as an intracellular messenger - inducing alterations in gene expression in tumors via the targeting of HIF-1 $\alpha$. As discussed in the text, a similar process of HIF- $1 \alpha$ stabilization by succinate occurs in LPSactivated macrophages, the process in this case being driven by signaling from TLR4 [4]. Figure 1, in main text, describes the parallels between tumors and LPS-activated macrophages in relation to succinate.

to repress inflammation and glycolysis, and to increase lipolysis, mitochondrial biogenesis, autophagy, and antioxidants (as recently reviewed [12]). Sirt1 deacetylates and inactivates the $\mathrm{p} 65$ subunit of $\mathrm{NF}-\kappa \mathrm{B}$, a key proinflammatory transcription factor [13]. Therefore, a decrease in $\mathrm{NAD}^{+}$, as demonstrated following LPS stimulation [4], would impair Sirt1 function and enhance NF-кB-dependent signaling. However, TLR4 stimulation has also been demonstrated to induce nicotinamide phosphoryltransferase-1 (Nampt1), an enzyme involved in $\mathrm{NAD}^{+}$synthesis, at later time-points, and this would enhance Sirt1 activity and thus dampen NF-кB-dependent responses, resulting in a negative-feedback effect. Sirt1 impaired the activity of another proinflammatory transcription factor, activating protein 1 (AP-1), in macrophages by targeting its subunits c-Jun and c-Fos [14], and thereby decreased transcription of the AP-1-dependent gene encoding cyclooxygenase 2 (COX2), an enzyme required for the synthesis of PGs. In a similar manner, Sirt1 deacetylates HIF-1 $\alpha$ and prevents its interaction with p300, a transcriptional coactivator required for its activity [15]. As discussed later, HIF-1 $\alpha$ is an important transcription factor that enhances glycolysis and the inflammatory response.

Sirt1 has also been shown to support the anti-inflammatory process, known as autophagy, which promotes the clearance of damaged organelles [16]. Sirt1 deacetylates autophagy-related gene products, resulting in their activation $[17,18]$, and regulates the quantity and quality of mitochondria [17]. Because mitochondria represent a source of ROS and therefore inflammation, Sirt1 may decrease ROS production from damaged mitochondria through activation of autophagy. Sirt1 further controls mitochondrial quality by activating peroxisome proliferator-activated 


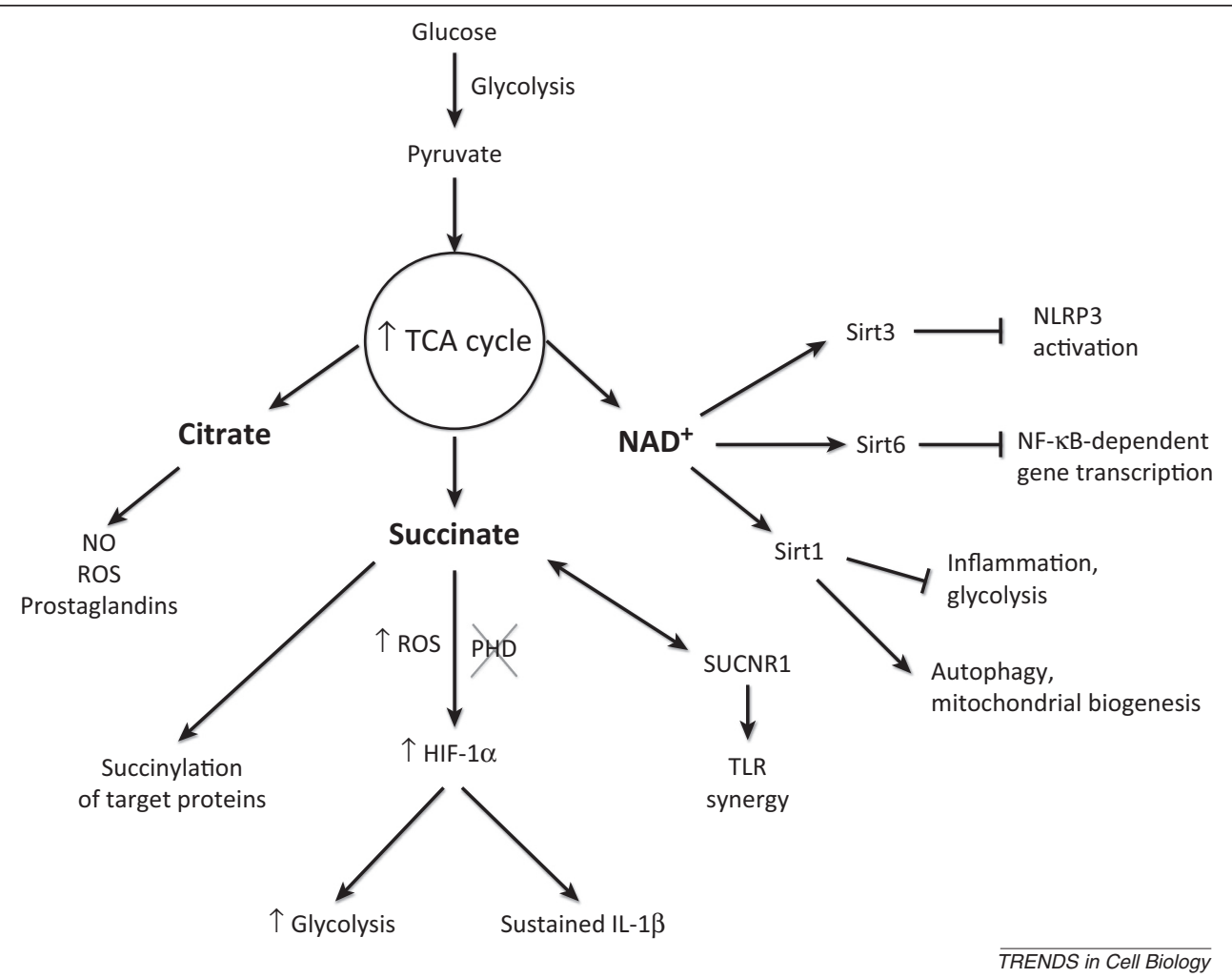

Figure 2. The function of tricarboxylic acid (TCA) cycle intermediates as inflammatory signals. Some metabolic intermediates of the TCA cycle have been demonstrated to act as inflammatory signals. Cytosolic citrate is required for fatty acid biosynthesis and is metabolized to produce reactive oxygen intermediates and prostaglandins, important inflammatory mediators. Succinate enhances glycolysis in several ways. It inhibits prolyl hydroxylase domain (PHD) enzyme function, both directly by product inhibition and indirectly via reactive oxygen species (ROS), driving hypoxia-inducible factor-1 $\alpha$ (HIF-1 $\alpha$ ) accumulation and increased glycolysis. ROS also inhibit mitochondrial function, boosting glycolysis as a result. Elevated HIF- $1 \alpha$ enhances the expression of genes containing HIF response elements (HREs), including the interleukin $1 \beta$ (IL-1 $\beta$ ) gene. Succinate can signal through succinate receptor 1 (SUCNR1) and act in synergy with Toll-like receptors (TLRs) to boost dendritic cell function. $N A D^{+}$exerts several anti-inflammatory effects by activating sirtuins, a class of $N A D^{+}$-dependent deacetylases. Sirt1 (sirtuin 1) inhibits the proinflammatory transcription factors nuclear factor $\mathrm{\kappa B}$ (NF- $\mathrm{KB}$ ), activator protein 1 (AP-1), and HIF-1 $\alpha$, and enhances autophagy-related gene function and oxidative metabolism. Sirt6 also impairs NF- $\mathrm{KB}$ dependent signaling by deacetylating target promoters. Finally, inactivation of Sirt3 has been demonstrated to enhance NLR family, pyrin domain containing 3 (NLRP3) inflammasome activation.

receptor $\gamma$ coactivator $1 \beta$ (PGC-1 $\beta$ ), which is a crucial transcription factor required for mitochondrial biogenesis [19]. Sirt1-dependent PGC-1 $\beta$ activation increases oxidative phosphorylation, and this may contribute to a net antiinflammatory effect by decreasing the number of damaged mitochondria and subsequent ROS production [9].

Decreased activity of another sirtuin, Sirt2, has also been implicated in inflammation. A recent study suggests a role for Sirt2 and $\mathrm{NAD}^{+}$in NLRP3 (NLR family, pyrin domain containing 3) inflammasome activation [20]. The authors demonstrated that a variety of activators of NLRP3 inhibit mitochondrial function and therefore limit $\mathrm{NAD}^{+}$concentrations and impair Sirt2 function. Sirt2 is responsible for the deacetylation of the microtubule component $\alpha$-tubulin, leading to its depolymerization. Therefore, decreased Sirt2 function results in the accumulation and polymerization of acetylated $\alpha$-tubulin. NLRP3 associates with the acetylated $\alpha$-tubulin and localizes to the mitochondria where it is activated in a ROS-dependent manner. Finally, Sirt6 has been demonstrated to deacetylate the promoter of specific NF-кB target genes including interleukin $1 \beta$ (IL-1 $\beta$ ), thereby limiting NF- $\mathrm{KB}$ accessibility to these promoters and decreasing transcription [21].

These results collectively suggest that sirtuins exert multiple mechanisms to dampen inflammation, and thereby represent an important class of regulatory enzymes whose functions are likely to be inhibited in cells treated with LPS, and possibly other inflammatory stimuli, given that LPS impairs $\mathrm{NAD}^{+}$accumulation.

\section{Succinate as an inflammatory signal}

In addition to citrate and $\mathrm{NAD}^{+}$, recent reports suggest that a third metabolite, succinate, can also act as a signal for inflammation [4]. Indeed, studies have shown that succinate can accumulate in immune cells, resulting in the stabilization of HIF- $1 \alpha$ or signaling via its receptor. We discuss these two functions of succinate in inflammation below.

\section{Succinate and HIF-1 $\alpha$ in inflammation}

The switch to glycolysis that occurs in activated macrophages is likely to be important at sites of inflammation where oxygen levels are low (hypoxia) [22], and HIF-1 $\alpha$ is central to this process. However, this switch can also occur under normoxia, with succinate acting as the signal. Under both situations, rapid ATP production and pentose-phosphate pathway activation occurs because of enhanced expression of HIF- $1 \alpha$-dependent genes. These events increase the biosynthetic capacity of the activated cell. An elevation in succinate had been reported in some 


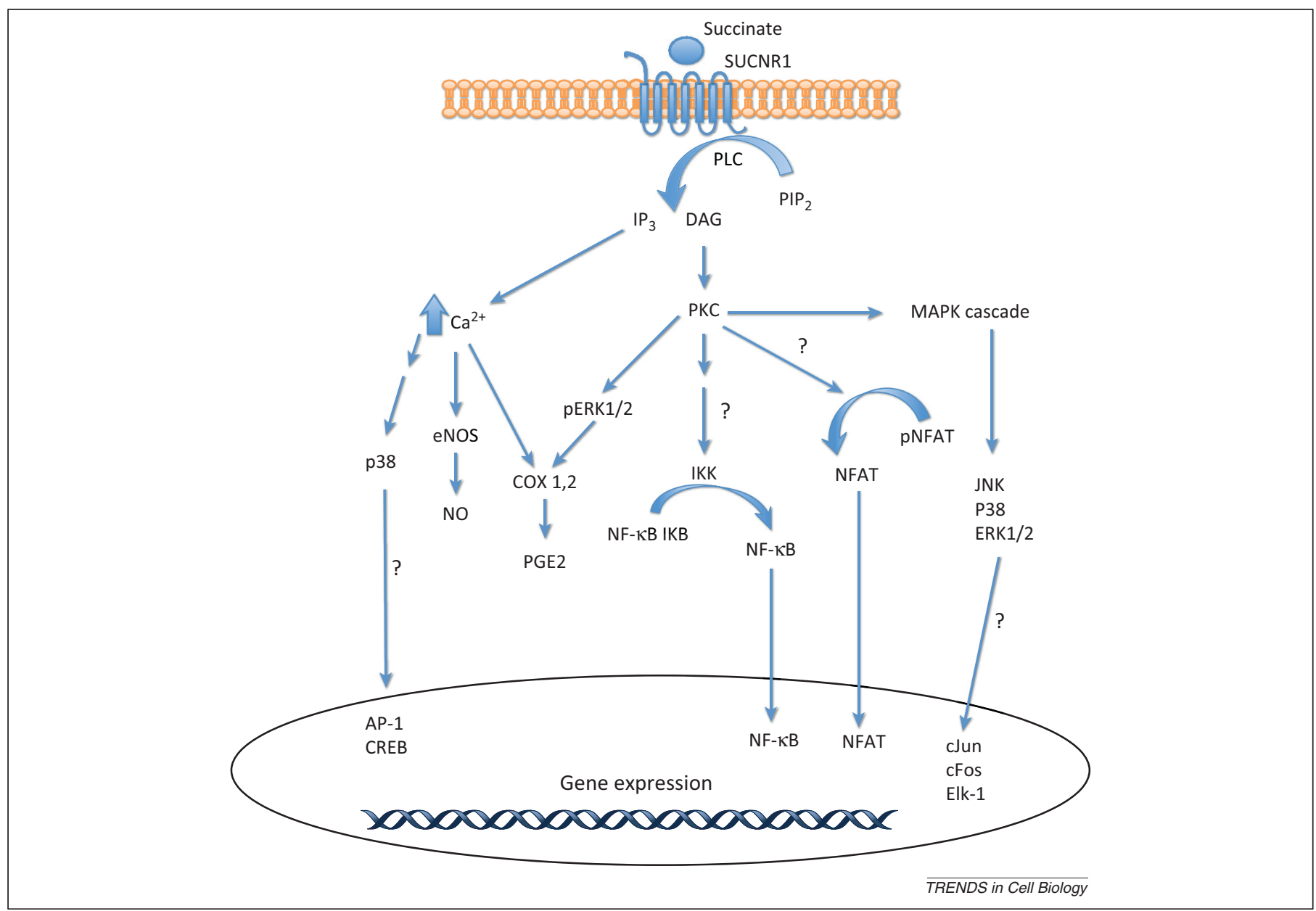

Figure 3. Succinate receptor 1 (SUCNR1) signaling. Succinate induces calcium mobilization in an adenylyl cyclase (AC) and protein kinase A (PKA)-dependent manner [45] SUCNR1 engagement activates phospholipase C (PLC), resulting in cleavage of phosphatidylinositol 4,5-bisphosphate (PIP2) to inositol trisphosphate (IP3) and

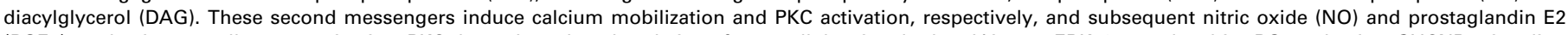
(PGE2) production as well as p38 activation. PKC-dependent phosphorylation of extracellular signal-related kinases ERK1/2 can also drive PG production. SUCNR1 signaling might act in synergy with several inflammatory signaling cascades. PKA is known to phosphorylate and activate the p65 subunit of nuclear factor $\kappa B$ (NF- $\mathrm{B}$ ) [46] and cAMP response element-binding protein (CREB). Furthermore, NF-kB, activating protein (AP)-1, nuclear factor of activated T cells (NFAT), and ETS domain-containing protein (Elk1) are all downstream targets of PKC and MAPKs. Question marks denote unexplored pathways following SUCNR1 ligation.

tumors, leading to stabilization of HIF-1 $\alpha$ (Box 1), and, recently, LPS-induced succinate was shown to act in a similar manner. Analogous to its effects in tumors, succinate was shown directly to inhibit prolyl hydroxylase domain (PHD) enzyme activity in macrophages, resulting in stabilization of HIF-1 $\alpha$ [4] and the induction of a range of target genes. Notably, the sustained induction of the proinflammatory cytokine IL- $1 \beta$ required HIF- $1 \alpha$. These data suggest that succinate may directly regulate $\mathrm{HIF}-1 \alpha$ signaling to promote inflammation by macrophages. This signaling could occur under normoxia because elevated succinate has the same effect on HIF-1 $\alpha$ stability as hypoxia (Figure 1).

In addition to inhibiting PHDs directly, succinate may also indirectly stabilize HIF-1 $\alpha$ by inducing ROS. It has been demonstrated that pharmacologic inhibition of succinate dehydrogenase (SDH), the enzyme responsible for the conversion of succinate to fumarate, or RNA interference of subunit B of SDH, induces HIF-1 $\alpha$ stabilization in a ROSdependent manner [23]. ROS can affect HIF-1 $\alpha$ by oxidizing $\mathrm{Fe}^{2+}$, a critical PHD cofactor, to $\mathrm{Fe}^{3+}$. As a result, PHD activity is limited, resulting in the indirect increase of HIF$1 \alpha$ stabilization [24].
In addition to LPS-treated macrophages, HIF-1 $\alpha$ activation has been detected in other inflammatory contexts. It is elevated in macrophages in the inflamed synovium in rheumatoid arthritis [25], and this may be important for the induction of IL-1 $\beta$ involved in synovial inflammation. HIF-1 $\alpha$ enhances differentiation of Tymphocytes into the proinflammatory Th17 subtype [7] by inducing IL-17 production and by enhancing the transcription of RAR-related orphan receptor $\gamma \mathrm{t}$ (ROR $\gamma \mathrm{t}$ ), the key transcription factor required for Th17 cell development. Whether succinate is needed for the Th17 response has yet to be determined. Interestingly, HIF-1 $\alpha$-deficient DCs show impaired ability to activate T cells [26], suggesting that this transcription factor is important for $\mathrm{T}$ cell polarization and activation. HIF- $1 \alpha$ therefore enhances inflammation by influencing both the innate and adaptive immune system and succinate may be crucial in both cases via its effect on HIF- $1 \alpha$ stabilization.

Succinate and succinate receptor 1 (SUCNR1) signaling during inflammation

Another function for succinate in inflammation is as the ligand for the G protein-coupled receptor (GPCR) GPR91, 
since renamed SUCNR1 (Figure 3). This indicates that succinate has a signaling role beyond HIF- $1 \alpha$ stabilization $[27,28]$. SUCNR 1 is highly expressed in mouse kidney, liver, spleen, small intestine [27], and on DCs [8]. The signaling cascade activated by SUCNR1 has only recently emerged [29] (Figure 3). Human embryonic kidney (HEK) 293 cells stably expressing SUCNR1 were stimulated with succinate, resulting in inositol trisphosphate (IP3) accumulation, calcium mobilization, and extracellular signalregulated kinase (ERK) phosphorylation [27] similar to Gi and Gq signaling cascades. Indeed, the use of the respective inhibitors of $\mathrm{Gq}$ and $\mathrm{Gi}$ signaling, YM254890 and pertussis toxin, impaired succinate-induced responses [30]. Calcium-dependent NO and prostaglandin E2 (PGE2) production was also observed following stimulation of SUCNR1 with succinate. In addition to HEK293 cells, succinate induces calcium mobilization and ERK1/2 phosphorylation in human monocyte-derived DCs (MoDCs) [8], suggesting SUCNR1 signaling is also functional in immune cells.

A recent study showed that succinate acts as a chemokine, inducing MoDC migration in vitro [8]. DCs treated in the presence of succinate displayed enhanced migration to draining lymph nodes, demonstrating its chemotactic function. Furthermore, SUCNR1-deficient mice displayed a decrease in the migration of DCs to skin draining lymph nodes compared with wild type mice upon stimulation. Succinate also influences inflammatory cytokine production in DCs. Succinate was found to act in synergy with the TLR3 ligand poly(I:C) and the TLR7 ligand imiquimod, increasing tumor necrosis factor $\alpha(\mathrm{TNF}-\alpha)$ production and expression [8]. Furthermore, IL-1 $\beta$ expression was enhanced in murine bone marrow-derived dendritic cells (BMDCs) treated with succinate and LPS simultaneously. Succinate also increased the capacity of DCs to act as antigen-presenting cells, which induce an adaptive response, thereby further exacerbating inflammation. When DCs were primed with succinate and antigen simultaneously, elevated antigen-specific $\mathrm{T}$ cell activation was observed, as demonstrated by increased TNF- $\alpha$ and interferon $\gamma$ (IFN- $\gamma$ ) production from these cells [8]. This effect was abrogated in SUCNR1-deficient DCs, confirming that succinate signaling is required for enhanced antigen-presenting function of these cells.

Recent findings from in vivo studies also support the role of SUCNR1 in mediating succinate-dependent inflammation. Skin grafts from SUCNR1-deficient mice showed prolonged allograft survival compared with wild type mice, which is likely due to the inability of DCs in the donor tissue to induce a host $\mathrm{T}$ cell response [8]. These results suggest that SUCNR1-deficient mice have an impaired immune response, perhaps because of decreased DC migration, reduced inflammatory mediator production, and subsequent impaired adaptive immune responses. This indicates that succinate accumulation and SUCNR1 signaling can profoundly influence immunity and inflammation.

Based on these studies, SUCNR1 antagonists might protect against exacerbated inflammation, such as that observed during graft rejection or autoimmunity. A selective antagonist for human and rat has been successfully

\section{Box 2. The role of succinate in disease}

The importance of succinate-induced SUCNR1 signaling has been demonstrated in disease states including hypertension and type 2 diabetes. NO and PGE2 are produced following SUCNR1 ligation and are classic drivers of renin release, an enzyme involved in regulating blood pressure, and a key component of the reninangiotensin system (RAS). Blocking NO and PGE2 production, using specific inhibitors of endothelial NOS and COX-2 respectively, limited succinate-induced renin release [47]. Furthermore, SDH inhibition following malonate treatment enhanced renin release, possibly as a result of succinate accumulation. Mice overexpressing renin developed glucose intolerance, and angiotensin II generated in response to renin impaired insulin receptor signaling and glucose transport [48]. In vivo succinate administration induced hypertension, but this did not occur in SUCNR1-deficient mice, which have diminished renin accumulation [27]. Finally, circulating succinate was increased in spontaneously hypertensive rats, as well as in obese and diabetic rats [49] and this may contribute to disease by stimulation of the RAS. It is well understood that IL- $1 \beta$ production can contribute to insulin resistance and disease in type 2 diabetes [22], but the role of succinate-induced IL-1 $\beta$ production in this process remains to be investigated.

Succinate is also elevated in inflammatory bowel disease. In mice undergoing dextran sulfate (DSS)-induced colitis there is more succinate present in the caecum and feces $[32,36]$. Furthermore, succinate is a major product of Bacteroides. Germ-free mice infected with Bacteroides vulgatus showed increased caecal succinate and increased severity of intestinal inflammation following DSS-induced colitis compared with controls suggesting bacteria from this family may be the source of succinate in this model. Administration of succinate to rat colon causes erosion of the colonic mucosa [50]. This is mediated by increased infiltration of superoxide-producing polymorphonuclear cells into the mucosa [50], providing mechanistic evidence of succinate-induced disease. These studies all suggest a role for succinate, possibly derived from bacteria, in inflammatory bowel disease.

generated [31], but whether this antagonist impacts the immune response has yet to be tested. However, it was found to be protective in hypertension, a disease in which succinate is elevated (Box 2).

\section{Succinylation as a PTM}

Another consequence of succinate accumulation may involve the PTM, lysine succinylation. Inhibition of SDH may result in the accumulation of succinate and lysine succinylation. Indeed, treatment of mouse fibroblasts with the SDH inhibitor 3-nitropropionic acid resulted in a slight increase in succinylation, suggesting that succinylation can be correlated with an increase in succinate [32]. This modification induces a $100 \mathrm{Da}$ change in mass, comparable to that of two well-established lysine modifications: acetylation and dimethylation. Importantly, it masks the positive charge on lysine, thereby likely resulting in a significant conformational change. Western blot analysis of whole cell lysates confirmed that this modification is evolutionarily conserved and possesses numerous substrates [33]. Succinylation might contribute to global cellular energy regulation because it appears that proteins involved in cellular metabolism are largely targets of succinylation [32]. Furthermore, succinylation is elevated in tissues of mice under fasting conditions [32]. These results suggest that succinylation might be an important response to the energy status of a cell - whereby it increases the activity of enzymes involved in glucose and lipid metabolism to meet cellular requirements for ATP [32]. 
Enzymes of the glycolytic pathway and TCA cycle, including glyceraldehyde 3-phosphate dehydrogenase (GAPDH) and isocitrate dehydrogenase (IDH) respectively, are targets of lysine succinylation [33], suggesting that this PTM may provide a method for enzymatic regulation. Mutation of Lys199 and Lys242, two established succinylation targets in IDH, decreased enzymatic activity, suggesting that these residues are functionally important and succinylation may affect enzymatic activity. Although the enzyme responsible for succinylation is yet to be identified, a potent desuccinylase (and demalonylase) has been uncovered [34]. Sirt5, another member of the NAD-dependent family of sirtuins, which was previously thought to function primarily as a deacetylase has been shown to have potent desuccinylase activity [34]. In support, Sirt5-deficient mice have increased succinylation at specific lysine residues of the enzyme carbamoyl phosphate synthase 1 (CPS1), a known target of succinylation. CPS1 has decreased activity in Sirt5-deficient mice suggesting that succinylation can impair enzymatic function [35]. Furthermore, several glycolytic enzymes were identified as targets of LPS-induced succinylation in macrophages. LPS decreases both Sirt5 expression and levels of $\mathrm{NAD}^{+}$in macrophages, while increasing protein succinylation, suggesting that LPS may regulate this PTM by decreasing Sirt5 [4].

Pyruvate dehydrogenase (PDH), a key metabolic enzyme responsible for the generation of acetyl-CoA from pyruvate that feeds the TCA cycle, is also a target of lysine succinylation [32]. Sirt5 was shown to desuccinylate PDH in mouse embryonic fibroblasts and decrease its enzymatic activity. In contrast to CPS1, succinylation may be required for PDH activity and Sirt5 may negatively regulate PDH. Similarly, the A subunit of SDH has also been shown to contain four lysine succinylation sites. Sirt5-deficient mice had significantly increased SDH activity, again suggesting that succinylation positively regulates its activity [32]. Furthermore, respiration is increased in Sirt5-deficient cells in the presence of pyruvate and succinate (but not ascorbate, the substrate of complex IV of the electron transport chain, ETC) [32]. This finding suggests that Sirt5 can target PDH and SDH specifically, repressing their function and inhibiting mitochondrial respiration, whereas succinylation enhances respiration. This conclusion is however in direct contradiction with literature demonstrating that LPS increases succinylation, decreases sirt5 and $\mathrm{NAD}^{+}$, and decreases respiration in mitochondria. Although this area requires further investigation, it is possible that these are time-dependent phenomena whereby an initial decrease in respiration occurs following LPS stimulation and at later time-points LPS-induced succinylation functions to restore mitochondrial respiration in the resolution phase of infection.

Although it is not clear how proteins are succinylated, it has been suggested that succinyl-CoA can passively attach to lysine residues, thereby succinylating these target sites [33]. Succinyl-CoA is in equilibrium with succinate, and this might explain the increased succinylation following LPS treatment, demonstrating a novel signaling function of succinate. Overall, protein succinylation is emerging as a PTM, which may be important for the metabolic changes occurring in inflammation.

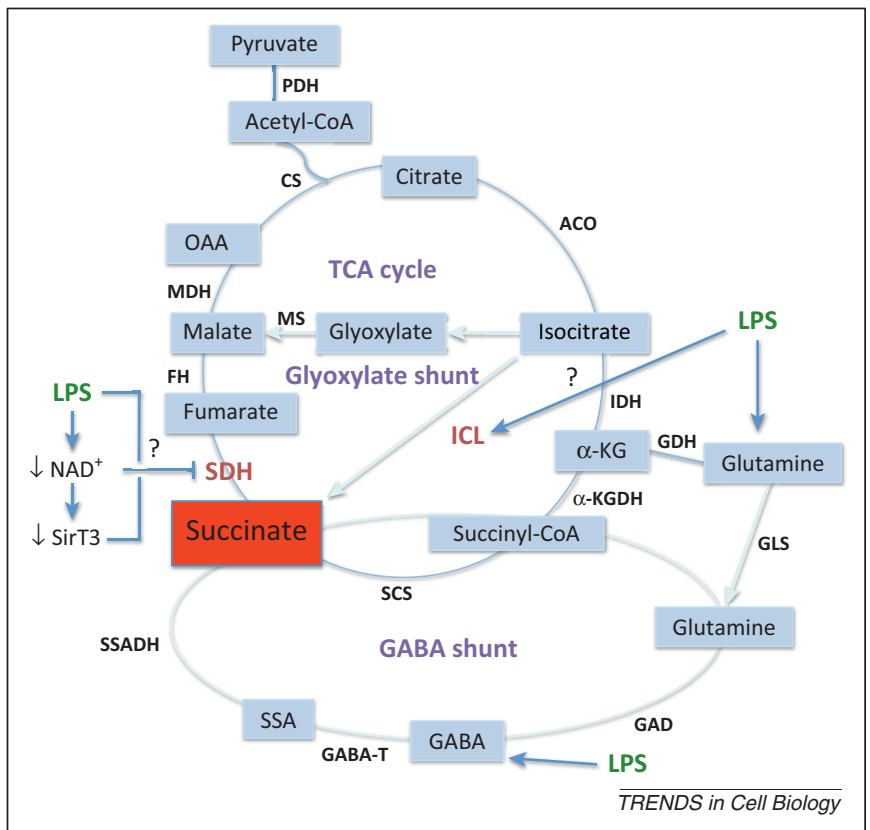

Figure 4. Possible mechanisms of LPS-induced succinate accumulation. Succinate is a byproduct of several metabolic pathways including the TCA cycle, the GABA shunt and the glyoxylate shunt. LPS has been shown to increase the expression of glutamine and GABA transporters and therefore cellular concentrations of these metabolites. Glutamine produces succinate through anapleurosis of $\alpha-K G$. LPS might increase the activity of ICL or decrease the activity of SDH to enhance succinate accumulation further. Abbreviations: ACO, aconitase; CS, citrate synthase; $\mathrm{FH}$, fumarate hydratase; GABA, $\gamma$-amino butyric acid; GAD, glutamate decarboxylase; GDH, glutamate dehydrogenase; GLS, glutaminase; ICL; isocitrate Iyase; IDH, isocitrate dehydrogenase; $\alpha-K G, \alpha$-ketoglutarate; $\alpha-K G D H, \alpha-K G$ dehydrogenase; LPS, lipopolysaccharide; MD, malate dehydrogenase; MS, malate synthase; OAA, oxaloacetate; $\mathrm{PDH}$, pyruvate dehydrogenase; SCS, succinyl-CoA synthetase; SDH, succinate dehydrogenase; SSA, succinic semialdehyde; SSADH, SSA dehydrogenase. TCA cycle, tricarboxylic acid cycle. Question marks denote unexplored pathways.

\section{Factors contributing to succinate accumulation}

We have so far discussed how the accumulation of succinate can promote inflammatory signaling; however, how succinate accumulates in the various immune cells is poorly understood. Recent studies on LPS-activated macrophages have begun to shed some light on the mechanisms regulating succinate accumulation. In macrophages treated with LPS, succinate production resulted from increased glutamine metabolism through anaplerosis of $\alpha$-ketoglutarate $(\alpha-\mathrm{KG})$ into the TCA cycle. In addition, LPS increased $\gamma$-aminobutyric acid (GABA), and its transporters, which can generate succinate via the 'GABA shunt' (Figure 4) - as shown by a reduction in LPS-induced succinate production after inhibition of GABA transaminase (GABA-T), a key enzyme of the GABA shunt, with vigabatrin.

Several other possible sources could contribute to the accumulation of succinate - including the glyoxylate shunt which converts isocitrate to succinate via the enzyme isocitrate lyase (ICL) (Figure 4). Although the majority of mitochondrial enzymes are inhibited by hypoxia [36], ICL is increased [37]. This may be to prevent membrane potential collapse by allowing succinate to feed complex II of the ETC. Supporting this, it has been shown that survival of Mycobacterium tuberculosis in hypoxic conditions is reduced when pathogen-derived succinate production is inhibited [38], suggesting that this metabolite is 
required for cell viability. Furthermore, compared to levels in resting macrophages, ICL activity was increased in $M$. tuberculosis-infected macrophages following activation with LPS [39], suggesting that LPS stimulation may increase the activity of this enzyme and therefore the production of succinate. Interestingly, whereas particular TCA cycle intermediates including succinate are elevated in macrophages following LPS stimulation, isocitrate and IDH are significantly decreased. This suggests that isocitrate is consumed independently of the TCA cycle, perhaps by the glyoxylate shunt [4] (Figure 4).

Decreased SDH activity may also be involved in succinate accumulation. SDH requires oxidized $\mathrm{FAD}$ and $\mathrm{NAD}^{+}$ as cofactors [40]. When present predominately in their reduced forms, such as during hypoxia, SDH function is impaired and succinate accumulates. LPS decreases $\mathrm{NAD}^{+}$ levels and may also affect FAD, thereby inhibiting SDH function. Decreased $\mathrm{NAD}^{+}$can affect the activity of Sirt3. Sirt3 has been shown to deacetylate SDHA, the active subunit of SDH, increasing its activity and thereby limiting succinate accumulation [40]. LPS decreases the expression of other members of the sirtuin family [4] and might also affect Sirt3. LPS has also been shown to impair SDH activity directly in African green monkey kidney cells [41]. It is clear that there are numerous methods of generating succinate in macrophages and that LPS stimulation may interfere at multiple levels, highlighting the importance of this metabolite for macrophage function (Figure 4).

\section{Concluding remarks}

The metabolites citrate, $\mathrm{NAD}^{+}$, and succinate are emerging as important signals in immunity and inflammation. This burgeoning area has highlighted numerous limitations, including our incomplete understanding of the role of these metabolite in vivo. In relation to succinate, the precise role of its receptor SUCNR1 in immunity needs to be explored further, and the role of succinylation as a PTM could lead to substantial new insights into cellular regulation. Finally, it is intriguing to speculate on whether targeting these processes might be useful in the clinic in situations where immunity requires boosting (in vaccines) or where immunity needs to be attenuated (in autoimmune and autoinflammatory diseases). The hope is that future studies will unveil the importance of these metabolic changes as signals in immune cell regulation, and this might in turn lead to novel therapeutic approaches.

\section{References}

1 Janeway, C.A., Jr and Medzhitov, R. (2002) Innate immune recognition. Annu. Rev. Immunol. 20, 197-216

2 Pearce, E.L. and Pearce, E.J. (2013) Metabolic pathways in immune cell activation and quiescence. Immunity $38,633-643$

3 Krawczyk, C.M. et al. (2010) Toll-like receptor-induced changes in glycolytic metabolism regulate dendritic cell activation. Blood 115, $4742-4749$

4 Tannahill, G.M. et al. (2013) Succinate is an inflammatory signal that induces IL-1beta through HIF-1alpha. Nature 496, 238-242

5 Everts, B. et al. (2012) Commitment to glycolysis sustains survival of NO-producing inflammatory dendritic cells. Blood 120, 1422-1431

6 Warburg, O. (1956) On the origin of cancer cells. Science 123, 309-314

7 Dang, E.V. et al. (2011) Control of T(H)17/T(reg) balance by hypoxiainducible factor 1. Cell 146, 772-784
8 Rubic, T. et al. (2008) Triggering the succinate receptor GPR91 on dendritic cells enhances immunity. Nat. Immunol. 9, 1261-1269

9 McGettrick, A.F. and O'Neill, L.A. (2013) How metabolism generates signals during innate immunity and inflammation. J. Biol. Chem. 288, 22893-22898

10 Infantino, V. et al. (2011) The mitochondrial citrate carrier: a new player in inflammation. Biochem. J. 438, 433-436

$11 \mathrm{He}$, W. et al. (2012) Mitochondrial sirtuins: regulators of protein acylation and metabolism. Trends Endocrinol. Metab. 23, 467-476

12 Liu, T.F. and McCall, C.E. (2013) Deacetylation by SIRT1 reprograms inflammation and cancer. Genes Cancer 4, 135-147

13 Yeung, F. et al. (2004) Modulation of NF-kappaB-dependent transcription and cell survival by the SIRT1 deacetylase. EMBO J. 23, 2369-2380

14 Zhang, R. et al. (2010) SIRT1 suppresses activator protein-1 transcriptional activity and cyclooxygenase- 2 expression in macrophages. J. Biol. Chem. 285, 7097-7110

15 Lim, J.H. et al. (2010) Sirtuin 1 modulates cellular responses to hypoxia by deacetylating hypoxia-inducible factor 1alpha. Mol. Cell 38, 864878

16 Levine, B. et al. (2011) Autophagy in immunity and inflammation. Nature 469, 323-335

17 Jang, S.Y. et al. (2012) Nicotinamide-induced mitophagy: event mediated by high $\mathrm{NAD}^{+} / \mathrm{NADH}$ ratio and SIRT1 protein activation. J. Biol. Chem. 287, 19304-19314

18 Lee, I.H. et al. (2008) A role for the NAD-dependent deacetylase Sirt1 in the regulation of autophagy. Proc. Natl. Acad. Sci. U.S.A. 105, 3374 3379

19 Lin, J. et al. (2005) Metabolic control through the PGC-1 family of transcription coactivators. Cell Metab. 1, 361-370

20 Misawa, T. et al. (2013) Microtubule-driven spatial arrangement of mitochondria promotes activation of the NLRP3 inflammasome. Nat. Immunol. 14, 454-460

21 Schug, T.T. et al. (2010) Myeloid deletion of SIRT1 induces inflammatory signaling in response to environmental stress. Mol. Cell. Biol. 30, 4712-4721

22 Tannahill, G.M. and O'Neill, L.A. (2011) The emerging role of metabolic regulation in the functioning of Toll-like receptors and the NOD-like receptor Nlrp3. FEBS Lett. 585, 1568-1572

23 Guzy, R.D. et al. (2008) Loss of the SdhB, but not the SdhA, subunit of complex II triggers reactive oxygen species-dependent hypoxiainducible factor activation and tumorigenesis. Mol. Cell. Biol. 28, 718-731

24 Kietzmann, T. and Gorlach, A. (2005) Reactive oxygen species in the control of hypoxia-inducible factor-mediated gene expression. Semin. Cell Dev. Biol. 16, 474-486

25 Hollander, A.P. et al. (2001) Expression of hypoxia-inducible factor 1alpha by macrophages in the rheumatoid synovium: implications for targeting of therapeutic genes to the inflamed joint. Arthritis Rheum. 44, 1540-1544

26 Wobben, R. et al. (2013) Role of hypoxia inducible factor-1alpha for interferon synthesis in mouse dendritic cells. Biol. Chem. 394, 495-505

27 He, W. et al. (2004) Citric acid cycle intermediates as ligands for orphan G-protein-coupled receptors. Nature 429, 188-193

28 Wittenberger, T. et al. (2001) An expressed sequence tag (EST) data mining strategy succeeding in the discovery of new G-protein coupled receptors. J. Mol. Biol. 307, 799-813

29 Ariza, A.C. et al. (2012) The succinate receptor as a novel therapeutic target for oxidative and metabolic stress-related conditions. Front. Endocrinol. 3, 22

30 Robben, J.H. et al. (2009) Localization of the succinate receptor in the distal nephron and its signaling in polarized MDCK cells. Kidney Int. $76,1258-1267$

31 Bhuniya, D. et al. (2011) Discovery of a potent and selective small molecule hGPR91 antagonist. Bioorg. Med. Chem. Lett. 21, 3596-3602

32 Park, J. et al. (2013) SIRT5-mediated lysine desuccinylation impacts diverse metabolic pathways. Mol. Cell 50, 919-930

33 Zhang, Z. et al. (2011) Identification of lysine succinylation as a new post-translational modification. Nat. Chem. Biol. 7, 58-63

$34 \mathrm{Du}$, J. et al. (2011) Sirt5 is a NAD-dependent protein lysine demalonylase and desuccinylase. Science 334, 806-809

35 Nakagawa, T. et al. (2009) SIRT5 deacetylates carbamoyl phosphate synthetase 1 and regulates the urea cycle. Cell 137, 560-570 
36 Hawkins, B.J. et al. (2010) Mitochondrial complex II prevents hypoxic but not calcium- and proapoptotic Bcl-2 protein-induced mitochondrial membrane potential loss. J. Biol. Chem. 285, 26494-26505

37 Wayne, L.G. and Lin, K.Y. (1982) Glyoxylate metabolism and adaptation of Mycobacterium tuberculosis to survival under anaerobic conditions. Infect. Immun. 37, 1042-1049

38 Eoh, H. and Rhee, K.Y. (2013) Multifunctional essentiality of succinate metabolism in adaptation to hypoxia in Mycobacterium tuberculosis. Proc. Natl. Acad. Sci. U.S.A. 110, 6554-6559

39 McKinney, J.D. et al. (2000) Persistence of Mycobacterium tuberculosis in macrophages and mice requires the glyoxylate shunt enzyme isocitrate lyase. Nature 406, 735-738

40 Cimen, H. et al. (2010) Regulation of succinate dehydrogenase activity by SIRT3 in mammalian mitochondria. Biochemistry 49, 304-311

41 McGivney, A. and Bradley, S.G. (1979) Action of bacterial endotoxin and lipid A on mitochondrial enzyme activities of cells in culture and subcellular fractions. Infect. Immun. 25, 664-671

$42 \mathrm{Wu}, \mathrm{W}$. and Zhao, S. (2013) Metabolic changes in cancer: beyond the Warburg effect. Acta Biochim. Biophys. Sin. 45, 18-26

43 Selak, M.A. et al. (2005) Succinate links TCA cycle dysfunction to oncogenesis by inhibiting HIF-alpha prolyl hydroxylase. Cancer Cell 7 , $77-85$
44 Maxwell, P.H. et al. (1999) The tumour suppressor protein VHL targets hypoxia-inducible factors for oxygen-dependent proteolysis. Nature 399, 271-275

45 Aguiar, C.J. et al. (2010) Succinate modulates $\mathrm{Ca}^{2+}$ transient and cardiomyocyte viability through PKA-dependent pathway. Cell Calcium 47, 37-46

46 Oeckinghaus, A. et al. (2011) Crosstalk in NF-kappaB signaling pathways. Nat. Immunol. 12, 695-708

47 Toma, I. et al. (2008) Succinate receptor GPR91 provides a direct link between high glucose levels and renin release in murine and rabbit kidney. J. Clin. Invest. 118, 2526-2534

48 Ghanem, F.A. and Movahed, A. (2007) Inflammation in high blood pressure: a clinician perspective. J. Am. Soc. Hypertens. 1, 113-119

49 Sadagopan, N. et al. (2007) Circulating succinate is elevated in rodent models of hypertension and metabolic disease. Am. J. Hypertens. 20 1209-1215

50 Fukui, S. et al. (1997) Mucosal blood flow and generation of superoxide in rat experimental colitis induced by succinic acid. J. Gastroenterol. $32,464-471$ 